\title{
Association of changes in body mass index during earlier adulthood and later adulthood with circulating obesity biomarker concentrations in middle-aged men and women
}

\author{
J. Montonen • H. Boeing • E. Schleicher • A. Fritsche • \\ T. Pischon
}

Received: 23 November 2010 / Accepted: 21 February 2011 /Published online: 6 April 2011

(C) Springer-Verlag 2011

\begin{abstract}
Aims/hypothesis The objective of our study was to investigate whether changes in BMI during earlier adulthood are more strongly associated with levels of circulating obesity biomarkers in middle age than are BMI changes during later adulthood.

Methods The study included 1,612 participants from the European Prospective Investigation into Cancer and Nutrition (EPIC)-Potsdam Study. The associations of BMI changes based on recalled BMI for the age ranges 2540 years (earlier adulthood) and 40-55 years (later adulthood) with later biomarker levels were compared using a linear model, adjusted for BMI at age 25 years and conventional risk factors.

Results BMI changes during both time periods as well as BMI at age 25 years were significantly associated with circulating levels of adiponectin, $\gamma$-glutamyltransferase (GGT), alanine aminotransferase (ALT), high-sensitivity Creactive protein (hs-CRP) and HDL-cholesterol (HDL-C) in
\end{abstract}

Electronic supplementary material The online version of this article (doi:10.1007/s00125-011-2124-6) contains supplementary material, which is available to authorised users.

J. Montonen $(\triangle) \cdot$ H. Boeing $\cdot$ T. Pischon

Department of Epidemiology,

German Institute of Human Nutrition Potsdam-Rehbruecke,

Arthur-Scheunert-Allee 114-116,

14558 Nuthetal, Germany

e-mail: jukka.montonen@dife.de

E. Schleicher $\cdot$ A. Fritsche

Department of Internal Medicine IV, University of Tuebingen,

Tuebingen, Germany

T. Pischon

Molecular Epidemiology Group,

Max Delbrueck Center for Molecular Medicine (MDC),

Berlin-Buch, Germany both sexes, and of $\mathrm{HbA}_{1 \mathrm{c}}$ in women. However, BMI gain for the age range $25-40$ years was significantly more strongly associated with unfavourable levels of adiponectin, hs-CRP, HDL-C and $\mathrm{HbA}_{1 \mathrm{c}}$ in men and women, and of GGT and ALT in men $(p$ difference $<0.05)$ than BMI gain for the age range 40-55 years. The percentage change in biomarker levels per unit gain in BMI for the age range 25-40 years ranged from $0.81 \%\left(\mathrm{HbA}_{1 \mathrm{c}}\right)$ to $9.80 \%$ (hs-CRP) in men, and from $0.75 \%\left(\mathrm{HbA}_{1 \mathrm{c}}\right)$ to $14.7 \%$ (hs-CRP) in women, whereas for the age range $40-55$ years, values ranged from $-0.15 \%$ to $4.82 \%$ in men and from $0.25 \%$ to $7.06 \%$ in women.

Conclusions/interpretation The results support the hypothesis that an increase in BMI in earlier adulthood is more strongly associated with unfavourable circulating levels of obesity biomarkers later in life than is an increase in BMI in later adulthood.

Keywords Adiponectin · ALT - Biomarker B BMI gain · Body mass index · GGT · HDL-cholesterol · hs-CRP. Obesity
Abbreviations
ALT Alanine aminotransferase
EPIC European Prospective Investigation into Cancer and Nutrition
GGT $\quad \gamma$-Glutamyltransferase
HDL-C HDL-cholesterol
hs-CRP High-sensitivity C-reactive protein

\section{Introduction}

Obesity is a well-established risk factor for cardiovascular disease, type 2 diabetes and other chronic diseases, but the 
underlying mechanisms are not yet fully understood [1]. A number of biomarkers closely related to obesity have been proposed to be relevant for these associations, including markers of insulin sensitivity (e.g. adiponectin), glucose metabolism (e.g. $\mathrm{HbA}_{1 \mathrm{c}}$ ), inflammation (e.g. C-reactive protein [CRP]), dyslipidaemia (e.g. HDL-cholesterol [HDL$\mathrm{C}]$ ) and hepatic fat accumulation (e.g. $\gamma$-glutamyltransferase [GGT] and alanine aminotransferase [ALT]). Lifestyle and surgical intervention studies have shown that weight loss is strongly associated with improved insulin sensitivity and glucose metabolism, as well as with decreased levels of liver enzymes [2-4]. However, recent studies suggest that disease risk may depend not only on the degree of adiposity, but also on body weight fluctuations during specific stages in life. For example, it has been shown that weight gain in earlier adulthood (i.e. between 25 and 40 years of age) is related to a higher risk and earlier onset of type 2 diabetes than weight gain in later adulthood (i.e. between 40 and 55 years of age) [5]. Yet surprisingly little is known about the extent to which BMI history or weight changes during life predict circulating levels of obesity biomarkers beyond the degree of adiposity.

In the present population-based study we aimed to investigate whether changes in BMI during earlier adulthood (age 25-40 years) are more strongly associated with levels of circulating obesity biomarkers (including adiponectin, GGT, ALT, $\mathrm{HbA}_{1 \mathrm{c}}$, HDL-C and high-sensitivity CRP [hs-CRP]) in middle-aged men and women than changes in BMI in later adulthood (age 40-55 years).

\section{Methods}

The European Prospective Investigation into Cancer and Nutrition (EPIC)-Potsdam Study is part of EPIC, a multicentre prospective cohort study designed to investigate the association between nutrition, cancer and other chronic diseases [6, 7]. In Potsdam, Germany, a total of 27,548 individuals $(16,644$ women aged $35-65$ years and 10,904 men aged 40-65 years) from the general population were recruited between 1994 and 1998 [8]. The health examination included anthropometric measurements, a personal health interview, a health questionnaire and blood sampling. The presence of diabetes mellitus at baseline was evaluated by a physician using information on self-reported medical diagnoses, medication records and dieting behaviour. Informed consent was obtained from all participants, and approval was given by the Ethical Committee of the State of Brandenburg, Germany.

The present study was based on a sub-cohort of 2,500 individuals who were selected at random from the total EPIC-Potsdam study population as part of separate casecohort studies on biomarkers and risk of type 2 diabetes and cardiovascular diseases $[9,10]$. For these study participants, data on all biomarkers were available for 2,204 individuals, of whom 1,639 had data available on weight history (Fig. 1a). Finally, after further exclusion of individuals with implausible data on changes in BMI (i.e. an increase of $>25 \mathrm{~kg} / \mathrm{m}^{2}$ or a decrease of $>15 \mathrm{~kg} / \mathrm{m}^{2}$ ) during each weight-change period, a total of 1,612 men and women remained for analysis.

Body weight and height were measured during the health examination by trained members of staff following standardised procedures while the study participants were wearing only underwear and no shoes [11]. During the personal computer-based interview, the participants were asked to state their weight at ages 25 and 40 years [8]. A high degree of reproducibility of self-reported recalled body weight at ages 25 and 40 years between two interviews administered 1 year apart was observed in a sub-study of the EPIC-Potsdam Study. Reproducibility of weight recall as classification into recall error strata within $3 \mathrm{~kg}$ was $75.8 \%$ at age 25 years and $81.7 \%$ at age 40 years [12].

Red blood cell levels of $\mathrm{HbA}_{1 \mathrm{c}}$ were measured using HPLC according to the manufacturer's instructions (Tosoh,

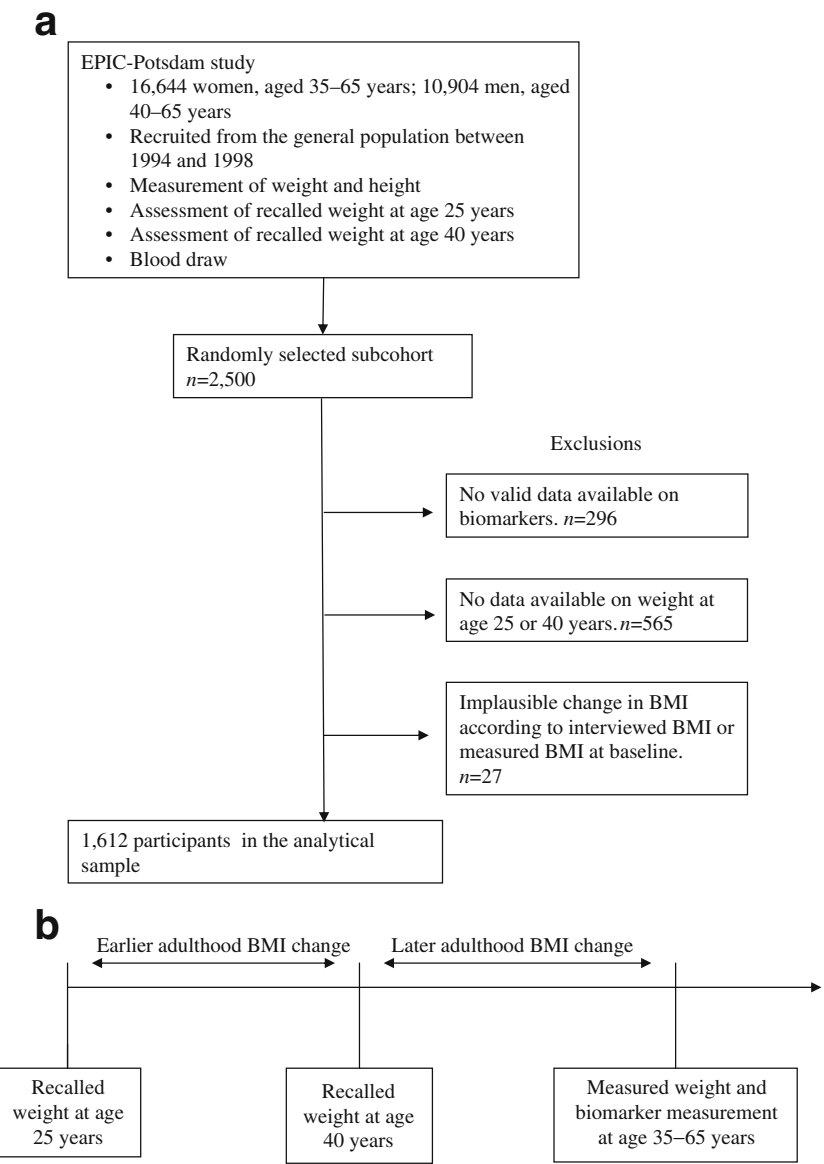

Fig. 1 a Reason for and number of excluded participants and (b) summary of weight history measurement 
Stuttgart, Germany). Plasma levels of ALT, GGT, HDL-C and hs-CRP were determined using the automatic ADVIA 1650 analyser (Siemens Medical Solutions, Erlangen, Germany). The total adiponectin concentration was measured by ELISA (Linco Research, St Charles, MI, USA) [13]. All assay procedures were performed as described by the manufacturer.

We calculated the change in BMI between the ages of 25 and 40 years and between the age of 40 years and age at the time of recruitment (Fig. 1b). Because the time period between age 40 years and the time of recruitment varied across the study participants and was on average shorter (median 13 years) than the 15-year period between the ages of 25 and 40 years, we estimated the BMI change for a standardised 15 -year period after age 40 years by linear regression [5]. The association between change in BMI and biomarker levels at the time of recruitment was investigated using general linear models. As a standard method in lifecourse epidemiology, BMI at recruitment was calculated as the sum of BMI at age 25 years, BMI change between the ages of 25 and 40 years, and BMI change between the age of 40 years and age at the time of recruitment [14]. Since the distributions of the levels of circulating biomarkers were right-skewed, log-transformed values were used in all analyses, and the strength of the associations was expressed as the percentage change in biomarker values by one unit change in the BMI component. In further analyses, categories of BMI change during each 15-year time period were formed as follows: (1) loss or stable (loss or gain of $<1 \mathrm{~kg} / \mathrm{m}^{2}$ over 15 years); (2) moderate gain (gain of 1.0 $4.0 \mathrm{~kg} / \mathrm{m}^{2}$ ); (3) severe gain (gain of $>4.0 \mathrm{~kg} / \mathrm{m}^{2}$ ). We then cross-tabulated these categories and calculated the adjusted geometric means with $95 \%$ CI of biomarker levels. Analyses were adjusted for age, sex, BMI at age 25 years (continuous), education (in or no training, vocational training, technical school, or technical college or university degree), occupational activity (light, moderate or heavy), sports activity $(0,0.1-4$ or $>4 \mathrm{~h} /$ week $)$, cycling $(0,0.1-2.4$, $2.5-4.9$ or $\geq 5 \mathrm{~h} /$ week), smoking (never, past, current $\leq 20$ cigarettes/day or current $>20$ cigarettes/day) and alcohol intake $(0,0.1-5,5.1-10,10.1-20,20.1-40$ or $>40 \mathrm{~g} /$ day $)$. In sensitivity analyses we restricted the population to the 642 men and 885 women who had no history of diabetes at the time of recruitment (based on self-reported diagnosis, medication use or dietary treatment), and who were aged 50-65 years at the time of recruitment. All analyses were performed separately for men and women. We evaluated potential effect modifications by sex of the association between BMI change and circulating biomarker levels by modelling interaction terms for sex and BMI change categories. All statistical analyses were performed using SAS release 9.1 (SAS Institute, Cary, NC, USA). All statistical tests were two- sided, and $p$ values $<0.05$ were considered to be statistically significant.

\section{Results}

The mean BMI at age 25 years was $23.5 \mathrm{~kg} / \mathrm{m}^{2}$ in men and $22.2 \mathrm{~kg} / \mathrm{m}^{2}$ in women. The personal characteristics of the participants at the time of recruitment according to BMI categories at age 25 years are presented in Table 1. Men with BMI $>30 \mathrm{~kg} / \mathrm{m}^{2}$ at age 25 years tended to report slightly higher levels of sports activity at recruitment than men with lower BMI levels (Table 1), whereas women with BMI $>30 \mathrm{~kg} / \mathrm{m}^{2}$ at age 25 years tended to report less sports and biking activities than women with lower BMI levels. For both men and women, the percentage of individuals with a university degree and the mean alcohol consumption were lower among obese participants at age 25 years than in those with a lower BMI (Table 1). Of the biomarkers, higher plasma concentrations of GGT, hs-CRP and $\mathrm{HbA}_{1 \mathrm{c}}$ were observed among obese men and women than among their counterparts with a lower BMI. Between the ages of 25 and 40 years the average BMI increased by $1.9 \mathrm{~kg} / \mathrm{m}^{2}$ in men and by $1.7 \mathrm{~kg} / \mathrm{m}^{2}$ in women, whereas between the ages of 40 and 55 years the average BMI increased by $2.0 \mathrm{~kg} / \mathrm{m}^{2}$ in men and by $3.2 \mathrm{~kg} / \mathrm{m}^{2}$ in women.

To investigate whether the association of BMI changes with biomarker levels differs between early and later adulthood, we included BMI at age 25 years and BMI changes between the ages of 25 and 40 years and between the ages of 40 and 55 years simultaneously in one model, and adjusted for age, sports activity, biking, education, occupational activity, smoking and alcohol consumption. We analysed men and women separately, as a significant interaction between sex and BMI change between the ages of 25 and 40 years was observed for ALT and hs-CRP, as well as between sex and BMI change between the ages of 40 and 55 years for hs-CRP and $\mathrm{HbA}_{1 \mathrm{c}}$ ( $p$ interaction $<0.05$ ). We found that BMI at age 25 years as well as changes in BMI between 25 and 40 years of age and between 40 and 55 years of age were each significantly related to circulating levels of adiponectin, GGT, ALT, hs-CRP and HDL-C in men, and to circulating levels of adiponectin, GGT, ALT, hsCRP, HDL-C and $\mathrm{HbA}_{1 \mathrm{c}}$ in women (Table 2). In men, a change in BMI between 25 and 40 years of age was more strongly related to circulating biomarker levels than was a change in BMI between 40 and 55 years of age ( $p=0.04$ for adiponectin, $p=0.02$ for GGT, $p=0.003$ for ALT, $p=0.04$ for hs-CRP, $p=0.005$ for HDL-C, and $p<0.001$ for $\mathrm{HbA}_{1 \mathrm{c}}$ ). For example, adjusted for BMI at age 25 years, among men a one unit gain in BMI between ages 25 and 40 years was associated with $9.80 \%$ (95\% CI 5.49-14.1) higher hs-CRP levels, whereas a one unit gain in BMI between ages 40 and 
Table 1 Characteristics of analytical sample at the time of recruitment across the categories of BMI $\left(\mathrm{kg} / \mathrm{m}^{2}\right)$ at age 25 years in men and women

\begin{tabular}{|c|c|c|c|c|c|c|}
\hline \multirow[t]{2}{*}{ Characteristic } & \multicolumn{3}{|l|}{ Men $(n=686)$} & \multicolumn{3}{|l|}{ Women $(n=926)$} \\
\hline & $<25(n=533)$ & $25-29.9(n=135)$ & $\geq 30(n=18)$ & $<25(n=810)$ & $25-29.9(n=100)$ & $\geq 30(n=16)$ \\
\hline Age (years) & $53.0 \pm 7.46$ & $54.6 \pm 7.22$ & $54.7 \pm 7.12$ & $52.5 \pm 6.96$ & $55.2 \pm 7.56$ & $55.4 \pm 5.67$ \\
\hline BMI at age 25 years $\left(\mathrm{kg} / \mathrm{m}^{2}\right)$ & $22.6 \pm 1.54$ & $26.4 \pm 1.15$ & $33.0 \pm 3.09$ & $21.6 \pm 1.84$ & $26.6 \pm 1.31$ & $33.0 \pm 2.39$ \\
\hline BMI at age 40 years $\left(\mathrm{kg} / \mathrm{m}^{2}\right)$ & $24.7 \pm 2.45$ & $28.1 \pm 2.24$ & $32.0 \pm 4.66$ & $23.4 \pm 2.75$ & $28.0 \pm 3.04$ & $32.6 \pm 4.77$ \\
\hline BMI at age 55 years $\left(\mathrm{kg} / \mathrm{m}^{2}\right)$ & $26.5 \pm 4.84$ & $30.4 \pm 4.93$ & $35.4 \pm 10.4$ & $26.6 \pm 5.37$ & $31.8 \pm 6.86$ & $34.8 \pm 7.74$ \\
\hline Sports activity (h/week) & $0.99 \pm 1.86$ & $0.99 \pm 1.97$ & $1.19 \pm 1.85$ & $0.94 \pm 1.60$ & $0.74 \pm 1.38$ & $0.47 \pm 0.76$ \\
\hline Biking (h/week) & $1.71 \pm 2.52$ & $2.06 \pm 3.20$ & $1.69 \pm 2.49$ & $1.86 \pm 3.05$ & $1.64 \pm 2.97$ & $1.38 \pm 2.00$ \\
\hline Alcohol consumption (g/day) & $23.0 \pm 21.7$ & $20.3 \pm 19.2$ & $13.3 \pm 10.4$ & $8.14 \pm 9.55$ & $7.94 \pm 10.9$ & $5.75 \pm 5.80$ \\
\hline Current or former smoker (\%) & 71.7 & 69.6 & 77.8 & 35.1 & 45.0 & 56.3 \\
\hline University degree (\%) & 54.6 & 44.4 & 33.3 & 28.3 & 13.0 & 18.8 \\
\hline Heavy physical work (\%) & 10.5 & 14.1 & 5.56 & 2.84 & 7.00 & 0.00 \\
\hline Prevalent diabetes $(\%)$ & 4.50 & 11.1 & 27.8 & 3.09 & 14.0 & 12.5 \\
\hline Adiponectin $(\mu \mathrm{g} / \mathrm{ml})$ & $5.64(5.42-5.88)$ & $5.08(4.69-5.50)$ & $5.58(4.72-6.60)$ & $8.62(8.36-8.88)$ & $7.80(7.00-8.68)$ & $6.33(4.86-8.26)$ \\
\hline GGT (U/l) & $28.0(26.1-30.1)$ & $31.9(28.1-36.3)$ & $35.0(21.9-55.9)$ & $14.3(13.6-15.1)$ & $17.8(15.4-20.5)$ & $23.0(15.2-34.9)$ \\
\hline ALT (U/l) & $25.5(24.4-26.5)$ & $29.6(26.9-32.6)$ & $24.8(20.4-30.2)$ & $16.9(16.4-17.4)$ & $20.2(18.5-22.1)$ & $21.4(17.2-26.7)$ \\
\hline hs-CRP (mg/l) & $0.64(0.23-0.24)$ & $0.94(0.24-0.25)$ & $1.48(0.24-0.27)$ & $0.80(0.24-0.24)$ & $1.37(0.23-0.25)$ & $2.16(0.15-0.29)$ \\
\hline HDL-C (mmol/l) & $1.18(1.16-1.21)$ & $1.11(1.07-1.16)$ & $1.00(0.89-1.13)$ & $1.42(1.39-1.44)$ & $1.27(1.20-1.34)$ & $1.36(1.24-1.50)$ \\
\hline $\mathrm{HbA}_{1 \mathrm{c}}(\%)$ & $6.57(6.50-6.64)$ & $6.80(6.65-6.96)$ & $7.71(6.98-8.52)$ & $6.47(6.42-6.52)$ & $6.81(6.60-7.03)$ & $6.67(6.14-7.25)$ \\
\hline
\end{tabular}

Values are means $\pm \mathrm{SD}$, percentages for categorical variables and geometric means with $95 \% \mathrm{CI}$ for biomarker variables

55 years was associated with $4.82 \%(95 \%$ CI $2.59-7.05)$ higher hs-CRP levels $(p=0.04)$. In women, a change in BMI between the ages of 25 and 40 years was significantly more strongly associated with circulating levels of adiponectin $(p=0.006)$, hs-CRP $(p<0.001)$, HDL-C $(p<$
$0.001)$ and $\mathrm{HbA}_{1 \mathrm{c}}(p=0.004)$ than $\mathrm{BMI}$ gain between the ages of 40 and 55 years. In women, no significant difference between the age ranges in the strength of the association was observed for liver enzymes $(p=0.17$ for GGT, $p=0.32$ for ALT). Exclusion of individuals younger

Table 2 Adjusted percentage change $(95 \% \mathrm{CI})$ in biomarker values per one unit increment of BMI at age 25 years, and per unit gain in BMI for the age ranges $25-40$ and $40-55$ years

\begin{tabular}{|c|c|c|c|c|}
\hline Variable & BMI at age 25 years & $\begin{array}{l}\text { BMI gain between } 25 \\
\text { and } 40 \text { years of age }\end{array}$ & $\begin{array}{l}\text { BMI gain between } 40 \\
\text { and } 55 \text { years of age }\end{array}$ & $p$ value $^{\mathrm{a}}$ \\
\hline \multicolumn{5}{|l|}{ Men } \\
\hline Adiponectin & $-2.71(-4.08,-1.34)$ & $-3.41(-4.96,-1.85)$ & $-1.62(-2.43,-0.81)$ & 0.04 \\
\hline GGT & $4.27(1.91,6.62)$ & $5.38(2.71,8.05)$ & $1.67(0.28,3.05)$ & 0.02 \\
\hline ALT & $2.81(1.36,4.27)$ & $4.59(2.94,6.24)$ & $1.75(0.90,2.60)$ & 0.003 \\
\hline hs-CRP & $9.47(5.68,13.26)$ & $9.80(5.49,14.10)$ & $4.82(2.59,7.05)$ & 0.04 \\
\hline HDL-C & $-1.97(-2.73,-1.21)$ & $-1.91(-2.77,-1.04)$ & $-0.52(-0.97,-0.08)$ & 0.005 \\
\hline $\mathrm{HbA}_{1 \mathrm{c}}$ & $1.28(0.90,1.67)$ & $0.81(0.38,1.25)$ & $-0.15(-0.37,0.08)$ & $<0.001$ \\
\hline \multicolumn{5}{|l|}{ Women } \\
\hline Adiponectin & $-1.66(-2.72,-0.60)$ & $-3.38(-4.58,-2.18)$ & $-1.42(-2.05,-0.80)$ & 0.006 \\
\hline GGT & $2.99(1.20,4.78)$ & $4.37(2.35,6.38)$ & $2.73(1.68,3.79)$ & 0.17 \\
\hline ALT & $2.30(1.29,3.32)$ & $2.52(1.37,3.66)$ & $1.85(1.25,2.45)$ & 0.32 \\
\hline hs-CRP & $8.92(5.92,11.92)$ & $14.7(11.4,18.1)$ & $7.06(5.29,8.82)$ & $<0.001$ \\
\hline HDL-C & $-1.28(-1.87,-0.70)$ & $-2.44(-3.10,-1.79)$ & $-0.68(-1.02,-0.34)$ & $<0.001$ \\
\hline $\mathrm{HbA}_{1 \mathrm{c}}$ & $0.45(0.19,0.71)$ & $0.75(0.46,1.04)$ & $0.25(0.09,0.40)$ & 0.004 \\
\hline
\end{tabular}

Adjusted by modelling. Multivariate model included age, sports activity, biking, education, occupational activity, smoking and alcohol consumption as well as BMI at age 25 years, BMI gain between ages 25 and 40 years, and BMI gain between ages 40 and 55 years

${ }^{\text {a }}$ Difference between age ranges $25-40$ and $40-55$ years 
than 50 years or older than 64 years and those with diagnosed diabetes at the time of recruitment did not materially change the results (data not shown). However, in women, besides liver enzymes the difference between the age ranges $25-40$ and $40-55$ years did not remain significant for $\mathrm{HbA}_{1 \mathrm{c}}(p=0.22)$.

To shed light on the observed difference between time periods in the association of BMI change with biomarker values we cross-classified individuals on the basis of three categories of BMI change (loss or stable, moderate gain, and severe gain) in earlier adulthood and later adulthood. In general, higher geometric mean values of GGT, ALT, hs-CRP and $\mathrm{HbA}_{1 \mathrm{c}}$ and lower values for adiponectin and HDL-C were observed among individuals with severe BMI gain in both time periods compared with those with loss or stable or moderate weight gain in both time periods (Fig. 2a,b, and electronic supplementary material [ESM] Table 1). However, in both sexes, those with severe BMI gain in the age range 25-40 years but BMI loss or stable BMI in the age range $40-55$ years tended to have higher values of GGT and $\mathrm{HbA}_{1 \mathrm{c}}$ and lower levels of adiponectin and HDL-C than individuals with weight loss or stable weight between the ages of 25 and 40 years but severe weight gain between the ages of 40 and 55 years. For example, among men whose BMI increased severely between ages 25 and 40 years but was stable or decreased between ages 40 and 55 years, the geometric mean $\mathrm{HbA}_{1 \mathrm{c}}$ level was $7.05 \%$ (95\% CI 6.64\%-7.14\%), whereas in men with weight loss or stable weight between ages 25 and 40 years but severe weight gain between ages 40 and 55 years the geometric mean $\mathrm{HbA}_{1 \mathrm{c}}$ value was $6.69 \%(95 \%$

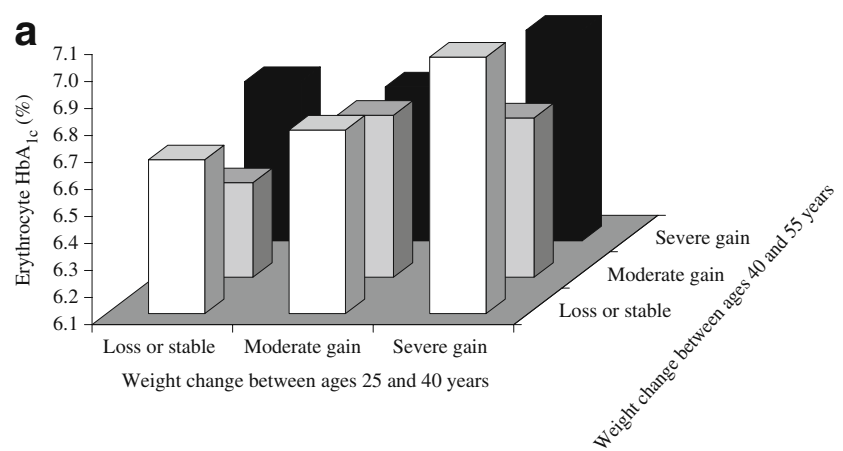

Fig. 2 Adjusted geometric mean values (adjusted for age, sports activity, biking, education, occupational activity, smoking and alcohol consumption and BMI at age 25 years) of $\mathrm{HbA}_{1 \mathrm{c}}(\%)$ according to categories of BMI changes between ages 25 and 40 years (on $x$-axis) and changes between ages 40 and 55 years (on $y$-axis) in (a) men and (b) women. In men, the geometric mean values across the increasing categories of weight gain between ages 40 and 55 years among those with a decrease in or stable BMI between ages 25 and 40 years were 6.67 (95\% CI 6.46-6.88), 6.45 (95\% CI 6.23-6.68) and 6.69 (95\% CI 6.38-7.01); among men with a moderate increase in BMI between ages 25 and 40 years the geometric mean $\mathrm{HbA}_{1 \mathrm{c}}$ values were 6.78 (95\% CI 6.57-6.99), 6.70 (95\% CI 6.50-6.92) and 6.67 (95\% CI 6.43-6.92); and among men with severe BMI gain between ages 25
CI $6.38 \%-7.01 \%$ ) when adjusted for age, BMI at age 25 years, sports activity, biking, education, occupational activity, smoking and alcohol consumption. Exclusion of individuals with a BMI of $30 \mathrm{~kg} / \mathrm{m}^{2}$ or more at age 25 years did not materially alter the results (data not shown).

\section{Discussion}

In the present study, BMI at age 25 years as well as BMI change in earlier adulthood (age range 25-40 years) and later adulthood (age range 40-55 years) were each significantly associated with circulating plasma levels of adiponectin, GGT, ALT, hs-CRP and HDL-C in middleaged men and women, and with $\mathrm{HbA}_{1 \mathrm{c}}$ in women. The association with circulating levels of obesity biomarkers in middle age was stronger for BMI change during earlier adulthood than for that during later adulthood for adiponectin, GGT, ALT, hs-CRP, HDL-C and $\mathrm{HbA}_{1 \mathrm{c}}$ in men, and for adiponectin, hs-CRP, HDL-C and $\mathrm{HbA}_{1 \mathrm{c}}$ in women. This suggests that BMI gain during earlier adulthood may be more important in determining these obesity biomarker levels than an increase in BMI later in life.

Obesity is a well-known risk factor for the development of insulin resistance, steatohepatosis and dyslipidaemia. The adipose tissue is capable of producing proinflammatory cytokines and hormones, which may systemically affect lipoprotein metabolism and insulin sensitivity [15]. In the present study, we examined biomarkers related to obesity that may be relevant for the development of

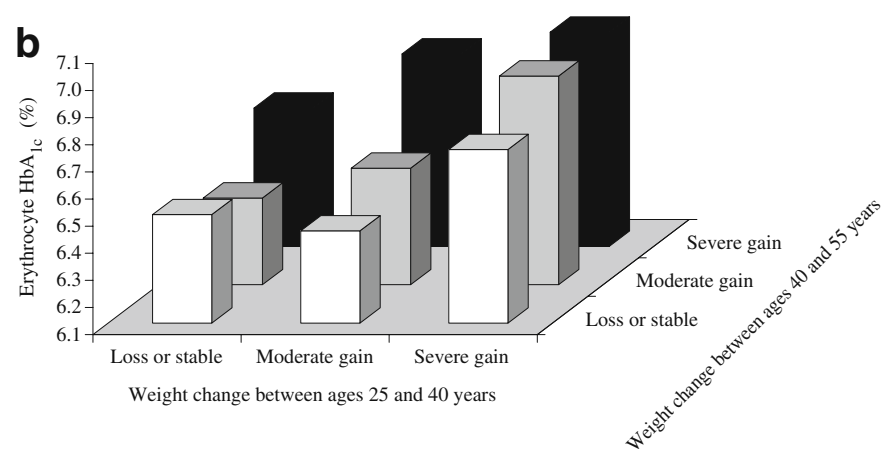

and 40 years they were 7.05 (95\% CI 6.74-7.37), 6.69 (95\% CI 6.28 7.13 ) and 6.88 (95\% CI 6.49-7.29). In women, the geometric mean values across the increasing categories of weight gain between ages 40 and 55 years among those with a decrease in or stable BMI between ages 25 and 40 years were 6.50 (95\% CI 6.33-6.69), 6.42 (95\% CI 6.25-6.60) and 6.61 (95\% CI 6.41-6.82); among women with moderate BMI gain between ages 25 and 40 years the corresponding geometric mean $\mathrm{HbA}_{1 \mathrm{c}}$ values were 6.44 (95\% CI 6.26-6.64), 6.53 (95\% CI 6.36-6.71) and 6.81 (95\% CI 6.63-7.01); and among women with severe BMI gain between ages 25 and 40 years $\mathrm{HbA}_{1 \mathrm{c}}$ values across the increasing categories of BMI change between ages 40 and 55 years were 6.74 (95\% CI 6.48-7.01), 6.87 (95\% CI 6.60-7.16) and 6.89 (95\% CI 6.64-7.14) 
chronic diseases, including markers of insulin sensitivity, glucose metabolism, inflammation, dyslipidaemia and hepatic fat accumulation. Several studies have suggested an association between an increase in BMI in adulthood and unfavourable lipid profiles [16-19] and onset of type 2 diabetes [20-30] as well as cardiovascular diseases after adjustment for attained weight at entry to the study [31-37]. However, although the association between obesity and risk of various chronic diseases is well documented, there is only limited information about the question of whether the time of onset of obesity or changes in BMI throughout life affects metabolism or disease risk beyond adiposity. The results of the present study suggest that an increase in BMI in earlier adulthood (age 25-40 years) may be more important for determining the metabolic profile related to disease risk at about 60 years of age than BMI gain in later adulthood. These results are consistent with the few previous studies that have simultaneously compared the associations between different weight history components and disease risk, including the risk of type 2 diabetes [5] and of metabolic syndrome [38]. Thus in the EPIC-Potsdam study it was previously shown that severe BMI gain in earlier adulthood was associated with a considerably higher (1.5 times higher in men and 4.3 times higher in women) diabetes risk compared with BMI gain in later life [5]. In the US National Health and Nutrition Examination Survey, weight gain between the ages of 25 and $40-54$ years suggested stronger associations with the presence of the metabolic syndrome as well as with lower plasma HDL-C and higher triacylglycerol values than did weight gain between the ages of 40-54 and 60 years [38]. Overall, these observations suggest that susceptibility to the development of diseases and abnormal metabolic profiles related to obesity may be time dependent, although the underlying mechanisms for such time dependency are unknown. Alternatively, one may speculate whether individuals who showed an increase in BMI in later adulthood may differ from those who gained weight in earlier adulthood in behavioural or genetic characteristics that may affect metabolism and risk profiles. For example, an increase in BMI in later adulthood may be more likely to be a result of a sedentary lifestyle, whereas BMI gain in earlier life may be more strongly related to genetic factors, and these factors may differ in their impact on metabolism and disease risk. In addition, the possibility cannot be ruled out that the longer duration of obesity among individuals whose BMI increased between the ages of 25 and 40 years is a significant factor contributing to metabolic disturbances reflected by biomarkers in the present study. Among women, one further explanation for the stronger association of the BMI increase during earlier adulthood with unfavourable circulating biomarker levels may be the childbearing years, which have been shown to be associated with the development of obesity [39-41]. However, a similarly stronger association of the BMI increase during earlier adulthood with unfavourable circulating biomarker levels was observed in men. Clearly, further studies are warranted to shed more light on the potential time dependency of the effects of obesity on metabolism, biomarkers and disease risk.

Some limitations of this study need to be considered. One limitation was that information on body weight at ages 25 and 40 years was self-reported, whereas weight and height at baseline were measured by trained staff. Although acceptable agreement between self-reported retrospective body weight and measured body weight has been shown $[42,43]$, it is possible that misclassification due to underreporting of past weight by overweight participants and overreporting by underweight participants may have occurred, which could influence the results. In the present study, we only had data available from a single blood sampling, which might have introduced random measurement errors in determining biomarkers. However, most of the markers presented here have been shown to have a suitable reliability for epidemiological studies [44, 45]. Circulating $\mathrm{HbA}_{1 \mathrm{c}}$ values tended to be slightly higher than expected. This may be due to the storage conditions for our blood samples. However, since the validity of the results on the association between exposure and outcome in epidemiological studies depends primarily on the ranking of individuals based on a biomarker rather than the absolute values, given that the ranking remains the same, the slightly overestimated $\mathrm{HbA}_{1 \mathrm{c}}$ levels do not affect the validity of our conclusions.

In summary, the present study demonstrated that among middle-aged men and women, an increase in BMI during earlier adulthood is more strongly associated with unfavourable circulating levels of obesity biomarkers than an increase in BMI during later adulthood. This suggests that metabolic susceptibility to changes in BMI varies according to age. The result is in line with studies which suggest that an increase in BMI in earlier adulthood is a stronger risk factor for diabetes or the metabolic syndrome than weight gain in later life [5, 38]. These results also support the hypothesis that a comprehensive history of weight gain in earlier adult life may provide a more precise risk assessment than a single measurement of BMI in later adulthood. Finally, the results of the present study highlight the importance of preventing weight gain in earlier adulthood.

Acknowledgements This study was partly supported by grants from the Federal Ministry of Education and Research, Germany (Bundesministerium für Bildung und Forschung, Förderkennzeichen 0315381A).

Duality of interest The authors declare that there is no duality of interest associated with this manuscript. The funding agency had no role in study design, data collection and analysis, decision to publish, or preparation of the manuscript. The responsibility for the content of this manuscript lies with the authors. 


\section{References}

1. Haslam DW, James WP (2005) Obesity. Lancet 366:1197-1209

2. Engl J, Bobbert T, Ciardi C et al (2007) Effects of pronounced weight loss on adiponectin oligomer composition and metabolic parameters. Obesity 15:1172-1178

3. Uusitupa M, Lindi V, Louheranta A, Salopuro T, Lindström J, Tuomilehto J (2003) Long-term improvement in insulin sensitivity by changing lifestyles of people with impaired glucose tolerance. Diabetes 52:2532-2538

4. Ueno T, Sugawara H, Sujaku K et al (1997) Therapeutic effects of restricted diet and exercise in obese patients with fatty liver. J Hepatol 27:103-107

5. Schienkiewitz A, Schulze MB, Hoffmann K, Kroke A, Boeing H (2006) Body mass index history and risk of type 2 diabetes: results from the European Prospective Investigation into Cancer and Nutrition (EPIC)-Potsdam study. Am J Clin Nutr 84:427-333

6. Riboli E, Hunt KJ, Slimani N et al (2002) European Prospective Investigation into Cancer and Nutrition (EPIC): study populations and data collection. Public Health Nutr 5:1113-1124

7. Boeing H, Wahrendorf J, Becker N (1999) EPIC-Germany-a source for studies into diet and risk of chronic diseases. European Investigation into Cancer and Nutrition. Ann Nutr Metab 43:195-204

8. Boeing H, Korfmann A, Bergmann MM (1999) Recruitment procedures of EPIC-Germany. European Investigation into Cancer and Nutrition. Ann Nutr Metab 43:205-215

9. Weikert C, Stefan N, Schulze MB et al (2008) Plasma fetuin-a levels and the risk of myocardial infarction and ischemic stroke. Circulation 118:2555-2562

10. Ford ES, Schulze MB, Bergmann MM, Thamer C, Joost HG, Boeing H (2008) Liver enzymes and incident diabetes: findings from the European Prospective Investigation into Cancer and Nutrition (EPIC)-Potsdam Study. Diabetes Care 31:1138-1143

11. Klipstein-Grobusch K, Georg T, Boeing H (1997) Interviewer variability in anthropometric measurements and estimates of body composition. Int J Epidemiol 26(Suppl 1):S174-180

12. Klipstein-Grobusch K, Kroke A, Boeing H (1998) Reproducibility of self-reported past body weight. Eur J Clin Nutr 52:525-528

13. Spranger J, Kroke A, Möhlig M et al (2003) Adiponectin and protection against type 2 diabetes mellitus. Lancet 361:226-228

14. De Stavola BL, Nitsch D, dos Santos SI et al (2006) Statistical issues in life course epidemiology. Am J Epidemiol 163:84-96

15. Wellen KE, Hotamisligil GS (2005) Inflammation, stress, and diabetes. J Clin Invest 115:1111-1119

16. Bot M, Spijkerman AM, Twisk JW, Verschuren WM (2010) Weight change over five-year periods and number of components of the metabolic syndrome in a Dutch cohort. Eur J Epidemiol 25:125-133

17. Hillier TA, Fagot-Campagna A, Eschwege E, Vol S, Cailleau M, Balkau B (2006) Weight change and changes in the metabolic syndrome as the French population moves towards overweight: the D.E.S.I.R. cohort. Int J Epidemiol 35:190-196

18. Rainwater DL, Mitchell BD, Comuzzie AG, VandeBerg JL, Stern MP, MacCluer JW (2000) Association among 5-year changes in weight, physical activity, and cardiovascular disease risk factors in Mexican Americans. Am J Epidemiol 152:974-982

19. Norman JE, Bild D, Lewis CE, Liu K, West DS (2003) The impact of weight change on cardiovascular disease risk factors in young black and white adults: the CARDIA study. Int J Obes Relat Metab Disord 27:369-376

20. Holbrook TL, Barrett-Connor E, Wingard DL (1989) The association of lifetime weight and weight control patterns with diabetes among men and women in an adult community. Int $\mathbf{J}$ Obes 13:723-729
21. Chan JM, Rimm EB, Colditz GA, Stampfer MJ, Willett WC (1994) Obesity, fat distribution, and weight gain as risk factors for clinical diabetes in men. Diabetes Care 17:961-969

22. Hanson RL, Narayan KM, McCance DR et al (1995) Rate of weight gain, weight fluctuation, and incidence of NIDDM. Diabetes 44:261-266

23. Colditz GA, Willett WC, Rotnitzky A, Manson JE (1995) Weight gain as a risk factor for clinical diabetes mellitus in women. Ann Intern Med 122:481-486

24. Wannamethee SG, Shaper AG, Walker M (2005) Overweight and obesity and weight change in middle aged men: impact on cardiovascular disease and diabetes. J Epidemiol Community Health 59:134-139

25. Brancati FL, Wang NY, Mead LA, Liang KY, Klag MJ (1999) Body weight patterns from 20 to 49 years of age and subsequent risk for diabetes mellitus: the Johns Hopkins Precursors study. Arch Intern Med 159:957-963

26. Ford ES, Williamson DF, Liu S (1997) Weight change and diabetes incidence: findings from a national cohort of US adults. Am J Epidemiol 146:214-222

27. Resnick HE, Valsania P, Halter JB, Lin X (2000) Relation of weight gain and weight loss on subsequent diabetes risk in overweight adults. J Epidemiol Community Health 54:596-602

28. Wannamethee SG, Shaper AG (1999) Weight change and duration of overweight and obesity in the incidence of type 2 diabetes. Diabetes Care 22:1266-1272

29. Sakurai Y, Teruya K, Shimada N et al (1997) Relationship between weight change in young adulthood and the risk of NIDDM. The Sotetsu study. Diabetes Care 20:978-982

30. Koh-Banerjee P, Wang Y, Hu FB, Spiegelman D, Willett WC, Rimm EB (2004) Changes in body weight and body fat distribution as risk factors for clinical diabetes in US men. Am J Epidemiol 159:1150-1159

31. Hubert HB, Feinleib M, McNamara PM, Castelli WP (1983) Obesity as an independent risk factor for cardiovascular disease: a 26-year follow-up of participants in the Framingham Heart study. Circulation 67:968-977

32. Manson JE, Colditz GA, Stampfer MJ et al (1990) A prospective study of obesity and risk of coronary heart disease in women. N Engl J Med 322:882-889

33. Manson JE, Willett WC, Stampfer MJ et al (1995) Body weight and mortality among women. N Engl J Med 333:677-685

34. Willett WC, Manson JE, Stampfer MJ et al (1995) Weight, weight change, and coronary heart disease in women. Risk within the 'normal' weight range. JAMA 273:461-465

35. Rimm EB, Stampfer MJ, Giovannucci E et al (1995) Body size and fat distribution as predictors of coronary heart disease among middle-aged and older US men. Am J Epidemiol 141:1117-1127

36. Galanis DJ, Harris T, Sharp DS, Petrovitch H (1998) Relative weight, weight change, and risk of coronary heart disease in the Honolulu Heart Program. Am J Epidemiol 147:379-386

37. Fulton JE, Shekelle RB (1997) Cigarette smoking, weight gain, and coronary mortality: results from the Chicago Western Electric study. Circulation 96:1438-1444

38. Alley DE, Chang VW (2010) Metabolic syndrome and weight gain in adulthood. J Gerontol A Biol Sci Med Sci 65:111-117

39. Smith DE, Lewis CE, Caveny JL, Perkins LL, Burke GL, Bild DE (1994) Longitudinal changes in adiposity associated with pregnancy. The CARDIA study. Coronary artery risk development in young adults study. JAMA 271:1747-1751

40. Williamson DF, Madans J, Pamuk E, Flegal KM, Kendrick JS, Serdula MK (1994) A prospective study of childbearing and 10year weight gain in US white women 25 to 45 years of age. Int J Obes Relat Metab Disord 18:561-569

41. Gunderson EP, Murtaugh MA, Lewis CE, Quesenberry CP, West DS, Sidney S (2004) Excess gains in weight and waist circumference associated with childbearing: The Coronary Artery 
Risk Development in Young Adults study (CARDIA). Int J Obes Relat Metab Disord 28:525-535

42. Stunkard AJ, Albaum JM (1981) The accuracy of self-reported weights. Am J Clin Nutr 34:1593-1599

43. Stevens J, Keil JE, Waid LR, Gazes PC (1990) Accuracy of current, 4-year, and 28-year self-reported body weight in an elderly population. Am J Epidemiol 132:1156-1163
44. Al-Delaimy WK, Jansen EH, Peeters PH et al (2006) Reliability of biomarkers of iron status, blood lipids, oxidative stress, vitamin D, C-reactive protein and fructosamine in two Dutch cohorts. Biomarkers 11:370-382

45. Pischon T, Hotamisligil GS, Rimm EB (2003) Adiponectin: stability in plasma over 36 hours and within-person variation over 1 year. Clin Chem 49:650-652 